\title{
A Importância da intergeracionalidade para desenvolvimento de universitários mais velhos
}

\section{The importance of intergenerationality to the development of older university students}

\section{La importancia de la intergeneracionalidad al desarrollo de los estudiantes universitarios de más edad}

\author{
Soniárlei Vieira Leite* \\ Universidade Salgado de Oliveira - UNIVERSO, Niterói, Rio de Janeiro, RJ, Brasil \\ Lucia Helena de Freitas Pinho França** \\ Universidade Salgado de Oliveira - UNIVERSO, Niterói, Rio de Janeiro, RJ, Brasil
}

\begin{abstract}
RESUMO
O envelhecimento tornou-se preocupação de diversas áreas do conhecimento, dentre elas o ensino-aprendizagem, com foco nas relações intergeracionais. Contudo, há uma carência de pesquisas que demonstrem os benefícios desta intergeracionalidade na educação universitária. Este trabalho teve por objetivo verificar a importância da intergeracionalidade no desenvolvimento dos estudantes mais velhos. A pesquisa teve abordagem qualitativa, por meio de entrevistas semiestruturadas e utilização da análise de conteúdo, baseadas na percepção de diferentes atores acerca da sabedoria, suporte social, habilidades sociais, superação (atitudes resilientes) e o contato intergeracional. Participaram desta pesquisa 20 universitários (10 mais jovens e 10 mais velhos), três professores e dois funcionários de duas universidades do Rio de Janeiro. $O$ contato intergeracional percebido é benéfico aos universitários, independentemente da idade, especialmente para os mais velhos, tanto para o seu desenvolvimento pessoal quanto para o desempenho acadêmico. A idade não é critério definidor do envelhecimento, nem na percepção dos universitários mais velhos, nem na dos mais jovens. As diferenças entre estudantes mais jovens e mais velhos, via de regra, são superadas ao longo do tempo. São recomendadas pesquisas sobre as habilidades sociais, especialmente as desenvolvidas no âmbito acadêmico, como fator de influência no desempenho dos universitários mais velhos.
\end{abstract}

Palavras-chave: intergeracionalidade, estudantes, jovens, velhos, desenvolvimento.

\begin{abstract}
Aging has become a concern in many areas of knowledge, among them the focus on teaching and learning in intergenerational relations. However, there is a lack of research demonstrating the benefits of this intergenerationality in university education. This study aimed to verify the importance of intergenerational development of older students. The research approach was
\end{abstract}


qualitative, through semi-structured interviews and use of content analysis, based on the perception of different actors about wisdom, social support, social skills, resilience (resilient attitudes) and intergenerational contact. The study gathered 20 students ( 10 younger and 10 older), three teachers and two staff from two universities of Rio de Janeiro. The intergenerational contact perceived is beneficial to the development of the university, regardless of age, but especially for the elderly. The intergenerational relationship is favorable to older students as their personal development and academic performance and age is not a defining criterion of aging on the perception of older and younger students. The differences between younger and older students, as a rule, are overcome over time. Researches on social skills are recommended, especially those developed in the academic, as an influence factor on the performance of older students.

Keywords: intergenerational, students, younger, older, development.

\section{RESUMEN}

El envejecimiento se ha convertido en una preocupación en muchas áreas del conocimiento, entre ellos el enfoque en la enseñanza y el aprendizaje en las relaciones intergeneracionales. Sin embargo, hay una falta de investigación que demuestra los beneficios de esta intergeneracionalidad en la educación universitaria. Este estudio tuvo como objetivo verificar la importancia del desarrollo intergeneracional de los estudiantes mayores. El enfoque fue cualitativa, a través de entrevistas y el uso de análisis de contenido semi-estructurados, basado en la percepción de los distintos actores sobre la sabiduría, el apoyo social, las habilidades sociales, la resiliencia (actitudes resilientes) y el contacto intergeneracional. El estudio reunió a 20 estudiantes ( 10 menores y 10 mayores), tres profesores y dos funcionarios de dos universidades de Río de Janeiro. El contacto intergeneracional percibido es beneficioso para el desarrollo de la universidad, independientemente de su edad, pero especialmente para las personas mayores. La relación intergeneracional es favorable a los estudiantes mayores como su desarrollo personal y el rendimiento académico y la edad no es un criterio de definición de envejecimiento en la percepción de los estudiantes de más edad y más jóvenes. Las diferencias entre los estudiantes más jóvenes y de mayor edad, por regla general, se superan con el tiempo. Se recomienda la investigación en las habilidades sociales, especialmente las desarrolladas en el ámbito académico, como un factor de influencia en el rendimiento de los estudiantes mayores.

Palabras clave: intergeneracional, estudiantes, joven, mayores, el desarrollo.

\section{I ntrodução}

Nas ultimas décadas, o envelhecimento da população brasileira deu um grande salto e tende a aumentar até 2040. De acordo com dados do Instituto Brasileiro de Geografia e Estatística (IBGE, 2013), o Brasil tinha 21 milhões de pessoas com idade igual ou superior a 60 anos em 2012, correspondente a $12,6 \%$ do total da população brasileira. A estimativa da Organização Mundial de Saúde (OMS, 2005) indica que o país será o sexto em número de idosos em 2025, quando provavelmente chegará ao patamar de 32 milhões de pessoas 
com 60 anos ou mais. Ao mesmo tempo em que as pessoas estão vivendo mais, há o grande desafio na garantia do bem-estar daqueles que envelhecem.

O crescimento do número de idosos é observado no mundo inteiro e a sua presença nas universidades tem sido constante (Freitas \& Scheicher 2010; Ruwer, Rossi \& Simon, 2005; Camarano et al., 1999), embora ainda haja pouca discussão sobre a sua inserção neste ambiente (Doll \& Stumpf Buaes, 2015; D'Alencar, 2002). O envelhecimento humano tornou-se objeto de estudo e preocupação de quase todas as áreas do conhecimento. O aprendizado para o idoso é fundamental para a continuidade das suas escolhas, autonomia e comunicar-se com o mundo.

Há um evidente interesse no conhecimento dos variados perfis das pessoas idosas e de como elas se percebem como elementos constitutivos da sociedade (Cachioni et al., 2014). Práticas como lazer, esportes, e retornar aos estudos, são atitudes que traduzem energia e força de superação, que favorecem a apreensão de conhecimentos novos e a autoestima. (Orth, Trzesniewski, \& Robins, 2010; Marques \& Pachane, 2010; Silveira et al., 2010). Estimular a participação dos idosos na sociedade é um dos pilares do envelhecimento saudável, segundo a OMS (2002). A inclusão social dos idosos é bem representada pelo ideal de sociedade justa de Uhlenberg (2000), que destaca a igualdade de participação de todos os atores sociais, para que não haja exclusão social, seja por ideologia, raça, sexo, religião e idade.

A educação é um dos instrumentos fundamentais para a inclusão social, vez que proporciona ao cidadão o sentimento de plenitude frente à capacidade de se expressar e exercer seus direitos, em todos os níveis e extratos sociais. Percebe-se que a importância da educação estende-se às pessoas mais velhas, na medida em que se verifica o aumento considerável de idosos nos bancos dos cursos de graduação das universidades brasileiras. Pimentel e Silva (2012) afirmam que o conhecimento empodera o idoso em sua tentativa de superar as dificuldades, ao mesmo tempo em que ele passa a exigir mais respeito, direitos e dignidade.

Ao compartilhar estudos e experiências com os mais jovens, os idosos se enriquecem e fazem enriquecer, reavaliando seus conceitos e preconceitos, em atitude que facilita a socialização. Essa relação intergeracional é valorizada sob a forma de solidariedade. A solidariedade intergeracional pode ser um fio condutor para a reversão de determinados valores, contribuindo para a ruptura de preconceitos, como o ageísmo, e promovendo um efeito positivo para a saúde e o bem-estar dos mais velhos (Antonucci, 2007). A solidariedade e o conflito interpessoal dominavam a literatura sobre intergeracionalidade até a década de 90 . Sob esta perspectiva, examinavam-se as características positivas ou negativas nos 
relacionamentos, de forma isolada (Bengtson, Giarrusso, Mabry, \& Silverstein, 2002).

A relação entre distintas gerações (intergeracionalidade) é estabelecida inicialmente pela família, por meio de apoio emocional, dos pais para com os filhos, dos netos com os avós. De acordo com a teoria da solidariedade, as relações intergeracionais variam em níveis de solidariedade afetiva, ou seja, em uma cadeia de sentimentos positivos entre pais e filhos. O conceito de intergeracionalidade emergiu da solidariedade, sugerindo que pais investem mais emocionalmente em suas relações do que os seus filhos (Bengtson \& Kuypers, 1971).

A relevância da solidariedade intergeracional no Brasil é abordada por França, Silva e Barreto (2010), demonstrando-se que o processo ensino-aprendizagem para os cidadãos mais velhos contribui para modificar a ideia preconceituosa da incapacidade apenas pela idade, bem como ajuda a incluir e reinseri-los no trabalho e na comunicação intergeracional. Isto não é tarefa fácil, sendo necessário que o planejamento para a integração intergeracional considere os interesses, alternativas, possibilidades e o contexto em que o cidadão mais velho e o mais jovem estão inseridos (Hogerbrugge \& Kornter, 2012; Goerres, \& Tepe, 2010).

A solidariedade intergeracional se faz sentir no segmento educacional, por seu compromisso com a prática pedagógica e a pesquisa, na psicologia social e nas pesquisas sobre as relações intergeracionais. Ela promove o desenvolvimento de todos os atores envolvidos, mas especialmente 0 do idoso; entendido 0 desenvolvimento como a capacidade de se manter independente 0 maior tempo possível, reforçando as relações de interdependência entre as gerações na família e na comunidade, além de manter a autonomia no caso dos fisicamente dependentes (Gorman, 1999).

A educação gerontológica contribui para a ciência do envelhecimento com a dimensão educativa na fase idosa da vida. O desenvolvimento do estudante mais velho, portanto, se dá dentro e fora do ambiente universitário, com a criação de programas que busquem um alto grau de satisfação e melhor qualidade de vida para os idosos envolvidos, bem como para os estudantes em geral. Facilitar a convivência e a experiência intergeracional, onde jovens e idosos desfrutem de um aprendizado mutuamente benéfico gera um impacto positivo na qualidade de vida. É um caminho eficaz para a integração social, um veículo para o enriquecimento cultural da população idosa e espaço de aprendizagem intergeracional para os jovens (González-Celis, Esquivel \& J iménez, 2005).

A promoção de uma nova perspectiva para o desenvolvimento psicológico no envelhecimento pode utilizar e estabelecer uma série de questões teóricas e metodológicas, que remetam ao desenvolvimento de práticas que contribuam para o desenvolvimento 
e bem-estar das pessoas mais velhas. A possibilidade de desenvolvimento permanente e bem-sucedido do idoso e a magnitude com que ocorrem as mudanças no envelhecimento são fatores que determinam o interesse de pesquisadores nos aspectos mais complexos do envelhecimento (Búfalo, 2013). O aperfeiçoamento do conhecimento e a efetividade das práticas direcionadas ao desenvolvimento psicológico no envelhecimento consubstanciam a análise dos caminhos percorridos pelos idosos, levando-se em consideração suas particularidades e individualidades; - que pode ganhar dimensões diversas com treinamento e aprendizagem (Brody, Chan, \& Caputi, 2010).

Em razão dessas diferenças, que se fazem sentir até mesmo pelo ambiente em que vivem, é que atitudes idênticas podem gerar comportamentos diferentes, de idoso a idoso. Logo, a chamada "ciência desenvolvimental" (Brofenbrenner, 2005) se constitui em abordagem fundamental e indispensável para a compreensão de sua contribuição para a discussão de sua promoção no desenvolvimento psicológico do indivíduo mais velho. Importante a determinação de uma visão sistêmica da Psicologia do Desenvolvimento (Tinajero \& Páramo, 2012).

O ensino-aprendizagem é um fator extremamente relevante como destacado por Janacsek, Fiser e Nemeth (2012), além de Aspen e Chapman (2000), independente da fase da vida de um cidadão, tornando-se mais um componente no quadro de fatores marcantes para a qualidade de vida dos mais velhos. A educação e a gerontologia andam de mãos dadas, quando o assunto é o idoso. A gerontologia, segundo Cachioni e Neri (2004), é uma área do conhecimento humano interdisciplinar voltado para ações, políticas e pesquisas com os mais velhos. Em termos normativos, o Estatuto do Idoso (Brasil, 2003) determina, ao Poder Público, a criação de oportunidades de acesso do idoso à educação, sendo necessária a adequação dos currículos, metodologias e material didático aos programas educacionais a ele destinados. Ainda de acordo com o Estatuto, o Poder Público apoiará a criação das Universidades Abertas da Terceira I dade e incentivará publicações adequadas aos idosos.

A divulgação do Estatuto do I doso representa a efetivação formal dos direitos deste segmento populacional. Não é a simples edição de uma lei que garante o exercício de direitos por parte do cidadão, mas a efetivação formal dos direitos é fundamental para o reconhecimento social dos mesmos direitos. Por meio do Estatuto houve o reconhecimento do conceito de igualdade material no tratamento equânime de todos os seres humanos, equiparando-os no que diz respeito às possibilidades de receber e desfrutar de oportunidades, pois todos devem possuir o mesmo grau de dignidade humana. $\mathrm{Na}$ igualdade material, as oportunidades devem ser oferecidas para 
todos, tanto na busca pela apropriação dos bens materiais, quanto na busca pelo aprimoramento espiritual.

$\mathrm{Na}$ Constituição Federal de 1988 encontram-se vários artigos que estabelecem normas programáticas, que objetivam reduzir as desigualdades existentes (por exemplo: art. 170 e incisos que tratam da ordem econômica e social; art. 205 que trata da democratização do ensino, dentre outros). A determinação do princípio da igualdade material no plano legislativo infraconstitucional objetiva dar efetividade prática às normas constitucionais, inclusive a referidas normas programáticas. O Estatuto do Idoso, lei federal n. 10.741 de 1 ㅇ. de outubro de 2003, prestigia esse princípio jurídico. Sarlet (2001) afirma que o princípio da igualdade baseia-se na dignidade da pessoa humana, sendo por essa razão que a Declaração Universal da ONU consagrou que todos os seres humanos são iguais em dignidade e direitos. Apesar de o Estatuto do Idoso estabelecer a idade de 60 anos para definir idoso brasileiro e determinar seus direitos fundamentais, é crucial lembrar que alguns direitos exigem dos idosos uma idade mais avançada, verbi gratia, o direito à gratuidade no transporte coletivo, que exige a idade mínima de 65 (sessenta e cinco) anos, conforme previsão do art. 230, § 2 o da Constituição Federal de 1988.

Uma vez que a educação é uma das molas propulsoras da qualidade de vida dos idosos (Boulton-Lewis, 2010), é de especial relevância não apenas a adoção de políticas públicas para a educação continuada (Bartholo, Rocha, Lima, \& Pacheco, 2012), mas a divulgação ampla dos direitos assegurados aos idosos, para que cidadãos de todas as faixas etárias tomem ciência da existência legal desta proteção aos mais velhos. Além disso, a realização de investigações sobre a sua inclusão e realidade nos mais diversos cenários da vida - educação, grupos sociais, no trabalho e outros podem sugerir intervenções para a redução de ocorrências contra eles (Pinto, Barham, \& Albuquerque, 2013).

Assim, este estudo investigou a importância da intergeracionalidade no desenvolvimento dos estudantes mais velhos. Paralelamente, foram investigados os motivos que levaram os estudantes a começar ou recomeçar os estudos - conforme a faixa etária; maiores dificuldades, benefícios e mudanças sentidas por ele sob a ótica do contato intergeracional. Finalmente, foi possível conhecer a percepção dos professores e funcionários acerca dos alunos mais velhos e seu desempenho em sala de aula.

$\mathrm{Na}$ revisão de literatura foram utilizados os descritores: intergeracionalidade e o desenvolvimento de idosos, entre 1990 e 2015. Os bancos de dados acessados foram Scielo, Google Acadêmico, ResearchGate, Latindex e, diretamente em revistas nacionais e internacionais da área de gerontologia. Foram encontrados 4.262 artigos e após leitura dos resumos foram 
selecionados 367 títulos, dos quais 118 considerados mais próximos à pesquisa. Deste total, selecionamos 37 títulos que mais aprofundaram a questão teórica e empírica da temática da intergeracionalidade no período supracitado. Não foi observado nenhum texto diretamente relacionado aos dois descritores supramencionados.

\section{Método}

A pesquisa utilizou a abordagem qualitativa, baseada em entrevistas semiestruturadas, buscando-se evidenciar a percepção dos diversos atores no âmbito universitário. As análises das narrativas de vida dos participantes, permitiu observar a dinâmica entre o mundo real e o mundo interior, objetivando alcançar a compreensão das crenças, atitudes, valores e motivações em relação aos comportamentos das pessoas em esferas sociais específicas. Assinala-se, ainda, que a finalidade da pesquisa qualitativa não é somente revelar opiniões ou pessoas, mas explorar o espectro dessas opiniões, bem como as diferentes representações sobre o assunto em questão (Bauer \& Gaskell, 2014).

\subsection{Participantes}

A amostra foi composta por 20 estudantes de dois grupos etários, ou seja, 10 idosos (com mais de 60 anos) e 10 jovens (entre 18 e 25 anos) oriundos de duas instituições privadas de Ensino Superior situadas na região metropolitana do Rio de Janeiro, selecionadas pelo número expressivo de estudantes mais velhos. Foram também entrevistados três professores (entre 45 e 50 anos) e dois funcionários (de 30 e 22 anos) que atuavam nas mesmas instituições universitárias.

\section{Seleção dos participantes}

Foram realizados contatos iniciais do pesquisador com as direções de cada instituição. O intuito desses contatos foi obter autorização das instituições e a identificação dos estudantes mais jovens e mais velhos, professores e funcionários que poderiam atender aos critérios básicos da pesquisa. A partir dessa identificação, os participantes foram convocados e marcados os dias das entrevistas.

A seleção dos alunos mais jovens e mais velhos foi realizada aleatoriamente nas duas universidades, tendo sido escolhidos pelo pesquisador um estudante mais jovem e um estudante mais velho. Já os professores e funcionários entrevistados foram pré-selecionados pelas direções universitárias que utilizaram os critérios de 
assiduidade e relacionamento próximo aos participantes jovens e idosos.

\subsection{Instrumento}

Foi adotada a técnica de entrevistas semiestruturadas, sendo elaborado um roteiro básico de perguntas para extrair as percepções dos participantes acerca do relacionamento entre as duas faixas etárias.

\section{Roteiro de Entrevista}

As entrevistas se deram, especialmente, como narrativas, ou seja, as perguntas foram formuladas sob a forma de intervenções sutis feitas ao longo da fala do participante, a partir da sua primeira manifestação. Foram iniciadas sempre com a pergunta de como ele se sentia na Universidade e como era o seu relacionamento com os seus colegas, tentando identificar nas falas as seguintes questões de forma a revelar a homogeneidade e a heterogeneidade dos alunos da Graduação:

1) Como se relacionam os estudantes mais jovens com os mais velhos, em sala de aula? Como eles se aproximam?

2) A aproximação entre eles interfere no desenvolvimento pessoal de ambos os grupos? De que forma se dá esta interferência?

3) Estudantes mais jovens e mais velhos se comportam de forma semelhante ou diferente, em sala de aula? Destaque alguns exemplos.

4) O comportamento influencia o desenvolvimento pessoal? Como se dá essa influência?

5) Você observa alguma relação entre a renda e o desenvolvimento dos estudantes. Como se verifica?

6) A formação educacional e a origem dos alunos (ensino médio público ou privado) podem influenciar na aproximação dos estudantes mais velhos com os mais jovens. De que forma se dá esta influência?

7) Esta mesma formação impacta nos resultados dos estudantes? Tais resultados são verificáveis?

8) A experiência profissional pode influenciar a convivência dos estudantes mais velhos com os mais jovens? De que maneira a experiência profissional influencia esta convivência?

9) A performance dos estudantes mais jovens e mais velhos está diretamente relacionada à sua experiência profissional? Como isto pode ser observado? 
10) A participação conjunta (mais velhos e mais jovens) em atividades de lazer e diversão pode influenciar o desenvolvimento de ambos?. Quais são as atividades desenvolvidas pelas duas gerações e como elas ocorrem?

\subsection{Procedimentos}

\subsubsection{Procedimentos éticos}

A pesquisa foi avaliada e aprovada pelo Comitê de Ética da Universidade, tendo os participantes concordado com o termo de consentimento livre e esclarecido.

\subsubsection{Procedimento de coleta de dados}

A coleta de dados levou aproximadamente três meses. No inicio das entrevistas, foi comunicado aos participantes que eles poderiam desistir da pesquisa a qualquer momento, que o convite era de aceitação voluntária, que não receberiam qualquer gratificação pela participação e os dados seriam confidenciais. Logo a seguir, foi solicitada a autorização pelos participantes para que as entrevistas fossem gravadas, bem como a assinatura do termo de consentimento livre e esclarecido. Foi evidenciado ainda que a assinatura do termo implicaria a autorização para que as informações obtidas nas entrevistas pudessem ser utilizadas exclusivamente para esta pesquisa e não haveria qualquer identificação dos participantes.

\subsubsection{Análise de dados}

As entrevistas foram baseadas nas variadas situações do dia-a-dia universitário, relatadas pelos participantes, sendo respeitados os critérios de saturação (Fontanella \& Magdaleno Júnior, 2012). Houve uma identificação apenas pelo grupo no qual os participantes estavam inseridos. No caso dos jovens de 18 a 25 anos, foi utilizado o código EJ ou estudante jovem (de 1 a 10). No caso das pessoas com 60 anos ou mais, foi utilizado o código El ou estudante idoso (de 1 a 10). No caso dos professores e funcionários foram utilizados os códigos $\mathrm{P}$ (de 1 a 3 ) e $\mathrm{F}$ ( 1 e 2 ), respectivamente. As entrevistas foram avaliadas por meio da "análise de conteúdo", estabelecendo-se a categorização das entrevistas.

A análise de conteúdo corresponde a um conjunto de técnicas de pesquisa cujo objetivo é a busca do sentido ou dos sentidos de um documento. Sendo um conjunto de técnicas de análise de comunicações, é fundamental a observância da semântica, entendida como a pesquisa do sentido de um texto, para a correta aplicação do método (Bauer \& Gaskell, 2014). 


\section{Resultados e discussão}

No contato inicial com os diretores das instituições de ensino, uma observação nos chamou atenção no tocante à seleção dos participantes por idade. Alguns diretores ficaram confusos se a idade de 60 anos seria o corte do grupo dos mais velhos, inclusive um deles questionou sua própria condição por ter mais de 60 anos. Ou seja, o velho é o outro, corroborando estudos anteriores que abordaram a definição de velho ou idoso (França \& Stepansky, 2005).

Neri e Cachioni (1999) afirmaram que a velhice e o envelhecimento são realidades distintas, uma vez que variam conforme a história, a cultura e a subcultura, as classes sociais, as histórias de vida, as condições educacionais, os estilos de vida, gênero, os grupos profissionais e as etnias. Neste estudo, optou-se pela definição sugerida pela OMS (2002) para os países em desenvolvimento, que fixou este marco temporal a partir de 60 anos.

Da análise de conteúdo das entrevistas foi efetuada a categorização, baseada no numero de vezes que um conceito, termo ou constructo era utilizado, na forma preconizada por Bardin (2013), conforme categorias relacionadas abaixo e descritas no Quadro 1 adiante.

Categorias e definições

a) Superação - atitudes de resiliência e autodeterminação no sentido de vencer obstáculos, conforme mencionado por Yunes (2003).

Os estudantes, professores e funcionários em geral apontaram a capacidade de superação dos mais velhos, em atitudes de persistência, determinação, força de vontade e, especialmente, resiliência. Psicologicamente resilientes são os idosos que não sucumbem aos problemas e adversidades (Fontes \& Neri, 2015). Estes idosos, em oposição às expectativas negativas de grande parte da sociedade, desenvolvem atitudes positivas e se adaptam ao ambiente que os cerca e se superam, demonstrando fortes indícios de resiliência. Resiliência é um conceito emprestado da física pela psicologia internacional (Rutter, 2006; Windle, Bennett \& Noyes, 2011) e no Brasil (Brandão, Mahfoud \& Gianordoli-Nascimento, 2011; Nalin \& França, 2015; Yunes, 2012).

Embora não haja consenso acerca do conceito, observa-se que os pesquisadores enfatizam a resiliência como um conjunto de atitudes positivas com o fito de enfrentar adversidades (Fourie \& Theron, 2012; Reppold, Mayer, Almeida, \& Hutz, 2012), sendo importante estudar a resiliencia no envelhecimento, vez que é um componente para o bem- estar. Atitudes de resiliência podem ser incorporadas em treinamentos, como reforço de comportamento assertivos em relação a situações de conflito (Nalin \& França, 2015). 
Unidade de sentido: i) “... os mais velhos são persistentes e mais determinados. Sempre entregam os trabalhos dentro do prazo..." (EJ6); ii) “... a gente tem mais dificuldade no aprendizado, mas não desistimos, temos que recuperar o tempo perdido, não dá pra ficar enrolando..." (EI3).

b) Sabedoria - experiência de vida e conhecimentos acumulados ao longo de uma vida produtiva, conforme mencionado por Baltes e Smith (1995).

O atributo "sabedoria" foi mencionado diversas vezes como fator influente de aproximação entre universitários mais jovens e mais velhos, tido como relevante para o desempenho acadêmico de ambos. Deve-se a Baltes (1939-2006) o entendimento do envelhecimento como um processo contínuo, de múltiplas dimensões e direções, marcado por perdas e ganhos. O desenvolvimento e o envelhecimento bem-sucedidos baseiam-se em três fatores: seleção de metas, otimização dos meios para atingir essas metas e compensação quando os meios disponíveis para atingir as metas não forem suficientes (Neri, 2006).

Os entrevistados relacionaram a sabedoria com a maturidade e a maior experiência de vida; o que pode ser um fator importante para transmissão de valores para os mais jovens. Creem que os mais velhos têm mais capacidade para superar os problemas pela experiência acumulada ao longo da vida. Acredita-se que seja possível repassar a sabedoria para o desenvolvimento pessoal dos mais jovens (DeMichelis, Ferrari, Rozin \& Stern, 2015).

Unidade de sentido: i) “... embora sejam lentos, eles têm a vantagem de ter mais experiência. Na minha turma têm dois que foram executivos..." (EJ9); ii) “... trazemos uma bagagem de vida e de trabalho que ajuda muito à molecada. O debate é bom e os professores gostam..." (EI 1); iii) “... é muito bom conviver com pessoas que têm muita coisa pra contar, especialmente quando falam dos trabalhos que fizeram. Isso ajuda no debate em aula..." (P3).

c) Contato intergeracional - relação entre pessoas de diferentes faixas etárias, conforme mencionado por Cachioni e Aguilar (2008).

\begin{tabular}{|c|c|c|}
\hline Partic. & $\begin{array}{l}\text { Idade Sexo } \\
\text { Categoria }\end{array}$ & Unidade de Sentido \\
\hline EJ1 & $\begin{array}{l}20 \\
4\end{array}$ & "... a gente se ajuda, não tem preconceito, todo mundo..." \\
\hline EJ2 & $\begin{array}{l}22 \\
6\end{array}$ & "... eles melhoram muito no final do curso, ficam mais..." \\
\hline EJ3 & $\begin{array}{l}22 \\
2\end{array}$ & "... conhecimento é poder eles já vêm com bastante..." \\
\hline
\end{tabular}




\begin{tabular}{|c|c|c|c|}
\hline$\overline{E] 4}$ & $\begin{array}{l}25 \\
3\end{array}$ & $\mathrm{~F}$ & "... no início não gostei, depois rolou, ficamos superamigas..." \\
\hline$\overline{\text { EJ5 }}$ & $\begin{array}{l}23 \\
5\end{array}$ & $\mathrm{~F}$ & "... Habilidoso, tinha jogo de cintura e se enturmou ..." \\
\hline EJ6 & $\begin{array}{l}18 \\
6\end{array}$ & $M$ & "... felizes quando conseguem trabalhar com o computador..." \\
\hline EJ6 & $\begin{array}{l}18 \\
1\end{array}$ & $M$ & "... os mais velhos são persistentes e determinados..." \\
\hline EJ7 & $\begin{array}{l}24 \\
5\end{array}$ & $\mathrm{~F}$ & "... impressionante como falava bem em público..." \\
\hline EJ8 & $\begin{array}{l}23 \\
3\end{array}$ & $M$ & "... mas depois que a gente se conhece tudo melhora..." \\
\hline$\overline{\text { EJ9 }}$ & $\begin{array}{l}19 \\
2\end{array}$ & $\mathrm{~F}$ & "... lentos, eles têm a vantagem de ter mais experiência..." \\
\hline EJ10 & $\begin{array}{l}23 \\
4\end{array}$ & $M$ & "... fiquei sabendo que ela tava também na Unati daqui..." \\
\hline EI1 & $\begin{array}{l}62 \\
6\end{array}$ & $\mathrm{~F}$ & "... Nós nos tornamos mais conscientes de nossos direitos..." \\
\hline$\overline{\text { EI1 }}$ & $\begin{array}{l}62 \\
2\end{array}$ & $\mathrm{~F}$ & "... trazemos uma bagagem de vida e de trabalho que..." \\
\hline$\overline{\mathrm{EI} 2}$ & $\begin{array}{l}63 \\
4\end{array}$ & $\bar{F}$ & "... foi importante passar pelo nivelamento, pois já não..." \\
\hline EI3 & $\begin{array}{l}60 \\
1\end{array}$ & $M$ & "... mas não desistimos, temos que recuperar o tempo..." \\
\hline EI4 & $\begin{array}{l}61 \\
4\end{array}$ & $\mathrm{~F}$ & "... a falta de apoio da minha familia fez ficar difícil..." \\
\hline EI5 & $\begin{array}{l}63 \\
5\end{array}$ & $\mathrm{~F}$ & "... lidar com os mais jovens, além de uma habilidade..." \\
\hline EI6 & $\begin{array}{l}63 \\
1\end{array}$ & $\mathrm{~F}$ & "... me esforcei muito pra chegar nessa universidade..." \\
\hline$\overline{\mathrm{EI7}}$ & $\begin{array}{l}66 \\
1\end{array}$ & M & "... tentei várias vezes até conseguir passar nessa matéria..." \\
\hline EI8 & $\begin{array}{l}65 \\
5\end{array}$ & $\mathrm{~F}$ & "... nós escrevemos melhor porque lemos mais que os jovens" \\
\hline$\overline{\text { EI9 }}$ & $\begin{array}{l}60 \\
6\end{array}$ & $\mathrm{~F}$ & "... tô muito melhor hoje, depois de todo esse tempo na..." \\
\hline EI10 & $\begin{array}{l}67 \\
3\end{array}$ & $M$ & "... mas dá pra conviver com boa vontade e tolerância..." \\
\hline$\overline{E I 10}$ & $\begin{array}{l}67 \\
4\end{array}$ & $M$ & "... mais fácil quando a Universidade nos oferece algum..." \\
\hline
\end{tabular}




\begin{tabular}{llll}
\hline P1 & 45 & $F$ & "... dos jovens, nossos alunos idosos também chegam lá..." \\
\hline P2 & 48 & F & "... há uma certa intolerância inicial, depois ficam amigos..." \\
& 4 & & \\
\hline P3 & 50 & M & "... conviver com pessoas que têm muita coisa para..." \\
\hline F1 & 30 & M & "... Não sei, não vejo muita diferença..." \\
\hline F2 & 22 & F & "... ela era bem tímida, mas ficou segura, durona, diferente..." \\
\hline
\end{tabular}

Legenda:: EJ - Estudante jovem; EI - Estudante idoso; P - Professor; F Funcionário.

M - Sexo masculino; F - Sexo feminino; Categoria 1 - Superação; Categoria 2 Sabedoria; Categoria 3 - Contato intergeracional; Categoria 4 - Suporte social; Categoria 5 - Habilidades sociais; Categoria 6 - Desenvolvimento pessoal.

Unidade de sentido: i) “... o papo não rola no início, mas depois que a gente se conhece tudo melhora..." (EJ 8); ii) “... é um aprendizado, o jeito de pensar é diferente, os costumes são outros, mas dá pra conviver com boa vontade e tolerância..." (EI10).

d) Suporte social - apoio recebido pela família, sociedade e programas específicos para acompanhamento do envelhecimento na universidade, conforme apontado por Antonucci e Akiyama (1987).

Vários foram os relatos da importância do suporte ou apoio social para o pleno desenvolvimento do universitário mais velho, sendo certo que há os que apresentam maior ou menor grau de dificuldade de adaptação e integração no ambiente acadêmico e de aprendizagem. Houve relatos de que aqueles que ingressaram na universidade com algum tipo de suporte (social ou familiar), via de regra, conseguiam melhores resultados na vida acadêmica. Também o apoio dos próprios estudantes, exercido por meio da solidariedade intergeracional (Santoro, 2014), apresenta diferentes padrões de suporte ao estudante idoso, sendo um facilitador para suas experiências acadêmicas. O envelhecimento bem-sucedido está associado ao adequado suporte social (Howie, Troutman-Jordan, \& Newman, 2014).

Unidade de sentido: i) “... a gente se ajuda, não tem preconceito, todo mundo é aluno..." (EJ 1); ii) “... foi importante passar pelo nivelamento, pois já tinha tempo que eu não abria um livro. Nesse ponto, a instituição me ajudou muito. Eu não tinha dinheiro pra pagar cursinho de português..." (El2).

e) Habilidades sociais - qualidades que permitam a interação social, a fim de satisfazer necessidades e expectativas, conforme apontado por Carneiro (2006).

As habilidades sociais trazidas pelos estudantes mais velhos, antes de ingressarem na universidade, foram reconhecidas pelos mais jovens e 
consideradas um diferencial para o desempenho acadêmico (Couto, Vanderbergue, Tavares, \& Silva, 2012; Tavares, Couto \& Silva, 2012). Tais habilidades sociais se manifestam em áreas e momentos variados, seja na vida pessoal e familiar ou no exercício de atividades laborais ou profissionais. Nos relatos dos entrevistados ficaram evidenciadas as habilididades sociais vividas antes da entrada dos estudantes na universidade (habilidades desenvolvidas no âmbito familiar, laboral ou profissional) e que, muitas das vezes, há a percepção de que essas habilidades fazem a diferença para o desempenho acadêmico. Não ocorreram relatos suficientes para se evidenciar que habilidades sociais também se desenvolveram ao longo do curso de graduação. Isso indica uma preocupação com a análise de fatores que possam mensurar os ganhos e as perdas das habilidades sociais ao longo da vida (Desjardins \& Warnke, 2012), onde se inclui a vida acadêmica.

Unidade de sentido: i) “... eu não via ele com um velhinho, era muito jovem de cabeça, muito habilidoso, tinha jogo de cintura e se enturmou rapidinho..." (EJ 5); ii) "... saber lidar com os mais jovens, além de uma habilidade, é um desafio para os coroas, mas tem sido muito bom, dá pra contribuir com jeitinho..." (EI5).

f) Desenvolvimento pessoal - crescimento individual enquanto participante de um grupo, conforme descrito por Neri (2006).

A percepção generalizada dos estudantes foi a de que o contato intergeracional contribuiu para o desempenho de ambos (jovens e velhos). Também se fez sentir o desenvolvimento no sentido amplo, como cidadãos. Independente do aspecto formal que envolve um curso de graduação, o sentir de aperfeiçoamento da cidadania foi observado. Mesmo os mais velhos reconheceram que o avançar no curso de graduação, a diversidade etária e a troca de experiência e valores os tornaram melhores cidadãos; o que corrobora os estudos de Faleiros (2007). Isto foi observado na fala de uma estudante idosa (El1): “... ninguém fica indiferente a cinco anos de graduação. Nós nos tornamos mais conscientes de nossos direitos, da cidadania...".

O crescimento das pesquisas acerca do desempenho acadêmico por influência familiar é notável (Roksa \& Potter, 2011). Entretanto, ainda são escassos os estudos que abordem a intergeracionalidade no ambiente universitário e seu impacto no desempenho acadêmico, entendido o desempenho acadêmico como um viés do desenvolvimento pessoal. Lacerda (2010) é um dos raros entusiastas com a temática. Contudo, ficou demonstrado que quanto mais positiva for a relação intergeracional, maior será o desempenho do universitário mais velho. Em vista da análise de conteúdo, nos relatos ficou evidenciada a percepção dos entrevistados de que os universitários mais velhos que recebem o apoio dos mais jovens costumam realizar melhor suas atividades acadêmicas, como afirmado pelo estudante jovem (EJ6): “... a gente faz trabalho em 
grupo com os mais velhos e eles ficam felizes quando conseguem trabalhar com o computador... “. I mportante notar que esta pesquisa previa analisar o desenvolvimento pessoal (objeto da pesquisa) embora nesta e em outras falas nos deparamos com o desempenho acadêmico. Tal constatação aponta para a necessidade de confirmar se o suporte social é de fato um preditor para o desempenho acadêmico (como apontado nas considerações finais, mais adiante).

Unidade de sentido: i) “... não sei dizer. Aqui na secretaria passa velhinha bem devagar, com muita dificuldade. Tem velhinho que é super agitado. Não sei, não vejo muita diferença..." (F1); ii) “... os jovens têm vantagem porque parece que já nasceram com o computador na cabeça, mas com dedicação e ajuda dos jovens nossos alunos idosos também chegam lá. Por outro lado, os resultados dos velhos costumam ser mais consistentes ..."(P1).

Confirmaram-se as impressões iniciais de que a relação intergeracional positiva é potencialmente favorável aos universitários mais velhos quanto ao seu desenvolvimento pessoal e desempenho acadêmico bem como que a idade não é critério para influenciar a competência. Os universitários e professores relataram que não há diferenças sensíveis de resultados (avaliações) entre as faixas etárias. Nos próximos estudos, já iniciados, em abordagem quantitativa, serão verificados comparativamente, dentre outros, os coeficienes de rendimentos dos alunos (jovens e idosos).

Sob a ótica dos universitários, independente da idade, eventuais diferenças e dificuldades de relacionamento são superadas ao longo do tempo. Também foi verificado que a separação entre os grupos não ocorria pela idade, mas utilizava o critério das habilidades sociais, postura e comportamento dos integrantes. Não era objetivo deste estudo essa verificação, mas a constatação deu-se pelos relatos apresentados. Os grupos se formavam, não pela idade, mas por vários critérios que traduziram as afinidades entre seus elementos, tais como as habilidades sociais, a forma como se posicionavam individual e conjuntamente (postura) com relação à convivência acadêmica e a própria forma de ser traduzida nos comportamentos pessoais. No entanto, alguns participantes jovens apresentaram uma visão estereotipada dos velhos - exemplo: rabugento, formal ou antiquado, da mesma forma que alguns idosos apresentaram atitudes mais modernas, sendo aceitos, inclusive como líderes.

Outro aspecto que nos chamou atenção na análise dos dados foi que o marco dos 60 anos parece não ser tão relevante no entendimento dos entrevistados. I sto nos remete o fato de que o conceito de velho, velhice ou envelhecimento depende dos contextos histórico, geográfico, psicossocial, político-econômico e social em que são analisados, sofrendo influencia sensível da Educação (França \& Soares, 2009). Assim, os resultados desta pesquisa devem ser considerados com parcimônia, pois existem diversos mitos e 
preconceitos que precisam ser estudados mais detalhadamente em pesquisas futuras.

Paralelamente, constatou-se que os professores e funcionários não estavam preparados para atuar como facilitadores da entrada do estudante mais velho. Não foi observado nos depoimentos destes profissionais qualquer preparação especial para o atendimento aos alunos mais velhos ou metodologia especifica por parte dos professores na abordagem ou fixação de conteúdo. Assim, outra recomendação advinda deste estudo é a organização de um treinamento para estes funcionários e professores por psicólogos da área de envelhecimento, de forma a facilitar e reforçar a inclusão destes alunos mais velhos e favorecer o desenvolvimento da solidariedade intergeracional e os benefícios que podem surgir para as duas faixas etárias.

Não foi verbalizado pelos estudantes a relevância no que diz respeito às habilidades sociais desenvolvidas ao longo da graduação; o que, sendo uma limitação, requer futuros estudos, visando a continuidade da presente pesquisa. Em razão das categorias identificadas, tal continuidade poderia abordar, de forma quantitativa, a relação do suporte social e das habilidades sociais no desempenho acadêmico dos universitários mais velhos.

Finalmente, não se observou a utilização de uma metodologia especial para lidar com o estudante mais velho, assim como os funcionários não se mostraram preparados especificamente para lidar com estudantes idosos, salvo alguns professores que, por iniciativa própria, resolveram repensar suas metodologias. Estes professores trabalharam na forma de seminários em que os mais velhos tiveram voz para compartilhar suas experiências de vida. Em parte, isso parece ocorrer porque os participantes idosos formam um grupo minoritário nas salas de aula. Isso seria uma justificativa para o fato de não haver conteúdos, instrumentos, materiais e cuidados ergonômicos voltados para melhor adaptação dos mais velhos nas salas de aula e a sua inclusão.

\section{Considerações finais}

Este estudo objetivou verificar a importância das relações intergeracionais no ambiente acadêmico para o desenvolvimento dos universitários. A abordagem qualitativa utilizou entrevistas semiestruturadas, tendo sido elaborado um roteiro como guia do pesquisador para a escuta dos alunos, professores e funcionários de duas instituições privadas de ensino superior, nos cursos de Psicologia e Direito.

As entrevistas foram submetidas à análise de conteúdo, respeitado o critério de saturação. No decorrer das entrevistas foram surgindo os 
atributos relativos à importância da intergeracionalidade sobre o desempenho acadêmico dos universitários mais velhos, destacandose: as experiências de vida e profissionais divididas com os mais jovens, as atitudes mais maduras diante de situações de conflito, a reflexão em contraponto a impulsividade dos mais jovens, a liderança pelo conhecimento acumulado, dentre outros. Pela observação reiterada nos relatos colhidos, as seguintes categorias foram selecionadas: a) superação; b) sabedoria; c) contato intergeracional; d) suporte social; e) habilidades sociais; e f) desenvolvimento pessoal.

Os resultados deste estudo sugerem que a intergeracionalidade encontrada nas universidades favorece o desenvolvimento dos universitários mais velhos e mais jovens em suas diversas facetas. Tanto os jovens quanto os idosos reconhecem que o contato intergeracional promove a renovação dos mais velhos, tendo em vista a necessidade de os mesmos acompanharem a velocidade da dinâmica da sociedade, que hoje é marcadamente influenciada pela tecnologia, pela virtualidade e por relações de trocas de informações, que são muito rápidas e efêmeras, mas que traduzem um momento histórico do qual o universitário mais velho é parte fundamental. Esta renovação possibilita maior expectativa quanto à continuidade das atividades dos mais velhos em todos os níveis e extratos sociais, e por mais tempo, já que, ao conviver com o mais jovem, permanece como elemento do grupo, mantendo sua identidade como um ser produtivo e participante. Uma vez que a diferença de idade não o alija do sentimento de pertencimento ao grupo social do qual faz parte, a inclusão social se estabelece e pode continuar a se desenvolver.

É preciso, contudo, continuar a investigar a contribuição da intergeracionalidade no que toca ao desempenho acadêmico, e esta é uma recomendação para futuras pesquisas. Poucos são os estudos acerca das relações entre intergeracionalidade e desempenho acadêmico, tanto na abordagem qualitativa quanto na quantitativa. Vale ainda reforçar a recomendação de Rabelo e Neri (2014) quanto à existência de um ambiente favorável às trocas entre gerações, que estimula atitudes positivas, reduzindo o preconceito pela idade e promovendo o equilíbrio cognitivo e emocional do estudante velho.

\section{Referências}

Antonucci, T. (2007). Social relations: an examination of Social Networks, Social Support, and Sense of Control. I A. T. C. Handbook of Psychology of Ageing. New York: Academic Press; 427-453. 
Antonucci, T., \& Akiyama, H. (1987). An examination of sex differences in social support among older men and women. Sex Roles, 17(11/12), 737-749.

Aspen, D., \& Chapman, J. (2000). Lifelong learning: concepts and conceptions. International Journal of Lifelong Education, 19(1), 2-19. doi: 10.1080/026013700293421.

Baltes, P., \& Smith, J. (1995). Psicologia da sabedoria: origem e desenvolvimento. In: Neri, A. Psicologia do envelhecimento tema selecionado na perspectiva do curso da vida. Papirus, 4172.

Bardin, L. (2013). L'Analyse de contenu (2e edition). Paris: Presses Universitaires de France.

Bartholo, M., Rocha, F., Lima, B.; \& Pacheco, L. (2012). Educação e Qualidade de Vida do Idoso: uma Ação de Extensão no Centro de Convivência do Idoso. Revista Fluminense de Extensão Universitária, 2(1), 21-32.

Bauer, M., \& Gaskell, G. (2014). Pesquisa qualitativa com texto, imagem e som: um manual prático. Petrópolis: Vozes.

Bengston , V., Giarrusso, R. , Mabry , J., \& Silverstein , M. (2002). Solidarity, conflict, and ambivalence: Complementary or competing perspectives on intergenerational relationships? Journal of Marriage and Family, 64, 568-576. doi: 10.1111/j.1741-3737.2002.00568.x

Bengtson, V., \& Kuypers, J. (1971). Generational difference and the developmental stake. International Journal of Aging and Human Development, 2, 249-260. doi: 10.2190/AG.2.4.b

Boulton-Lewis, G. (2010). Education and learning for the elderly: why, how, what. Education and learning for the elderly: why, how, what. Educational Gerontology, 36(3), 213-228. doi: $10.1080 / 03601270903182877$

Brandão, J., Mahfoud, M. \& Gianordoli-Nascimento, I. (2011). A construção do conceito de resiliência em psicologia: discutindo as origens. Paidéia, 21 (49), 263-271. Disponível em: http://dx.doi.org/10.1590/S0103-863X2011000200014

Brasil, Lei n. 10.741, de 1 o de outubro de 2003 (2003). Estatuto do I doso. Diário Oficial da União. Brasília, DF.

Brody, T., Chan, A., \& Caputi, P. (2008). Comparison of older and younger adult's attitudes towards and abilities with computers: implications for training and learning. British Journal of Educational Technology, 41(3), 473-485. doi: 10.1111/j.14678535.2008.00914.x

Bronfenbrenner, U. (2005). Making human beings human: Bioecological perspectives on human development. Thousand Oaks, CA: Sage. 
Búfalo, K. (2013). Aprender na terceira idade: educação permanente e velhice bem-sucedida como promoção de saúde mental do idoso. Revista Kairós Gerontologia, 16(2), 195-212.

Cachioni, M., \& Aguilar, L. (2008). A convivência com pessoas idosas em instituições de ensino superior: a percepção de alunos de graduação e funcionários. Revista Kairós, 11(1), 79-104.

Cachioni, M., \& Neri, A. (2004). Educação e gerontologia: desafios e oportunidade. RBCEH - Revista Brasileira de Ciências do Envelhecimento Humano, 99-115.

Cachioni, M., Ordonez, T., Silva, T., Batistoni, S., Yassuda, M., Melo, R., Domingues, M., \& Lopes, A. (2014). Motivational factors and predictors for attending a continuing education program for older adults. Educational Gerontology, 40(8), 584-596. doi: 10.1080/03601277.2013.802188.

Camarano, A. et al. (1999). Como vive o idoso brasileiro? In A. A. Camarano (Org.), Muito além dos 60: os novos idosos brasileiros (pp. 281-306). Rio de Janeiro: IPEA.

Carneiro. R. (2006). A relação entre habilidades sociais e qualidade de vida na terceira idade. Revista Brasileira de Terapias Cognitivas, 2(1), 45-54.

Couto, G., Vanderbergue, L., Tavares, W., \& Silva, R. (2012). Interações e habilidades sociais entre universitários: um estudo correlacional. Estudos de Psicologia, 29(supl.), 667-677.

D'Alencar, R. (2002). Ensinar a viver, ensinar a envelhecer: desafios para a educação de idosos. Estudos Interdisciplinares, 4, 61-83.

DeMichelis, C., Ferrari, M., Rozin, T., \& Stern, B. (2015). Teaching for wisdom in an intergenerational high-school-English class. Educational Gerontology, 41(8), 551-566. doi: 10.1080/03601277.2014.994355

Desjardins, R., \& Warnke A. (2012). Ageing and skills: a review and analysis of skill gain and skill loss over the lifespan and over time (OECD Education Working Papers, 72). Paris: OECD Publishing.

Faleiros, V. (2007). Cidadania e direitos da pessoa idosa. Ser Social (UNB), 20, 35-61.

Fontanella, B., \& Magdaleno Júnior, P. (2012). Saturação teórica em pesquisa qualitativa: contribuições psicanalíticas. Psicologia em Estudo, 17(1), 63-71.

Fourie, C. L., \& Theron, L. C. (2012). Resilience in the face of fragile $X$ syndrome. Qualitative Health Research, 22, 1355-1368. doi: $10.1177 / 1049732312451871$

Fontes, A., \& Neri, A. (2015). Resiliência e velhice: revisão de literatura. Ciência \& Saúde Coletiva, 20(5), 1475-1495. Disponível em: http://dx.doi.org/10.1590/141381232015205.00502014. 
França, L., Silva, A., \& Barreto, M. (2010). Programas intergeracionais: quão relevantes eles podem ser para a sociedade brasileira? Revista Brasileira de Geriatria e Gerontologia, 13(3), 519-532.

França, L. \& Soares, D. (2009). Preparação para a aposentadoria como parte da educação ao longo da vida. Psicologia, Ciência e Profissão, 29(4), 738-751.

França, L. \& Stepansky, D. (2005). Educação permanente para trabalhadores idosos - o retorno à rede social. Boletim Técnico do SENAC. 31(2), 47-55.

Freitas, M., \& Scheicher, M. (2010). Qualidade de vida dos idosos institucionalizados. Revista Brasileira de Geriatria e Gerontologia. 13(3), 395-401.

González-Celis, R., Esquivel, H., Jiménez, F. (2005). Impacto de un aula para personas mayores sobre la calidad de vida. Una experiencia inter-generacional. Avances de un proyecto. Revista Interamericana de Educación de Adultos. 27(1), 95-109.

Goerres, A., Tepe, M. (2010). Age-based self-interest, intergenerational solidarity and the welfare state: a comparative analysis of olde people's attitudes towards public children en 12 OECD countries. European Journal of Political Research, 49(6), 818-851. doi: 10.1111/j.14756765.2010.01920.x

Gorman, M. (1999). Development and the rights of older people. Earthscan Publications, 3-21.

Hogerbrugge, M., \& Kornter, A. (2012). Solidarity and ambivalence: comparing two perspectives on intergenerational relations using longitudinal panel data. The Journal of Gerontology, Series B. Psychological Sciences and Social Scienses. doi: 10.1093/geronb/gbr15

Howie, L., Troutman-Jordan, M., \& Newman, A. (2014). Social support and successful aging assisted living residents. Educational Gerontology, 40(1), 61-70. doi: 10.1080/03601277.2013.768085

Instituto Brasileiro de Geografia e Estatística - IBGE (2013). Síntese dos indicadores sociais, estudos e pesquisas. Rio de J aneiro.

Janacsek, K., Fiser, J., \& Nemeth, D. (2012). The best time to acquire new skills: age related differences in implicit sequence learning across the human lifespan. Developmental Science, 5(4), 496505. doi: $10.111 / \mathrm{j} .1467-7687.2012 .01150 . x$

Lacerda, W. (2010). Solidariedade entre irmãos e relações intergeracionais na construção de percursos escolares de excelência. Revista Educação em Perspectiva, 1(1), 82-102.

Marques, D., \& Pachane, G. (2010). Formação de educadores: uma perspectiva de educação de idosos em programas de EJA. Educação e Pesquisa, 36(2), 475-490. 
Nalin, C. \& França, L. (2015). A importância da resiliência para o bem-estar na aposentadoria. Paidéia, 25(61), 191-199. Disponível em: http://dx.doi.org/10.1590/198243272561201507.

Neri, A. (2006). O legado de Paul B. Baltes à Psicologia do Desenvolvimento e do envelhecimento. Temas em Psicologia, 14(1), 17-34. Disponível em: http://pepsic. bvsalud.org/scielo. php?script=sci_arttext\&pid=S1 413-389X2006000100005.

Neri, A., \& Cachioni, M. (1999). Velhice bem sucedida e educação. In A. Neri \& G. Debert (Org.), Velhice e sociedade (pp. 113-140). Campinas: Papirus.

Organização Mundial de Saúde (2002). Active aging: a policy framework. Geneva: OMS.

Organização Mundial de Saúde (2005). Envelhecimento ativo: uma política de saúde. Brasília: Organização Pan-Americana da Saúde.

Orth, U., Trzesniewski, K., \& Robins, R. (2010). Self-esteem development from young adulthood to old age: a cohortsequential longitudinal study. Journal of Personality and Social Psychology, 98(4), 645-658.

Pimentel, M., \& Silva, M. (2012). Inclusão social do idoso. In F. Pereira (Coord), Teoria e prática da gerontologia - um guia para cuidadores de idosos. Psicosoma (pp. 215-226). Disponível em: http://hdl. handle.net/10198/8887.

Pinto, F., Barham, E., \& Albuquerque, P. (2013). Idosos vítimas de violência: fatores sociodemográficos e subsídios para futuras intervenções. Estudos e Pesquisas em Psicologia, 13(3), 11591181.

Disponível em: http: // pepsic. bvsalud.org/scielo.php?script=sci_arttext\&pid=S1 808-42812013000300018.

Rabelo, D., \& Neri, A. (2014). A complexidade emocional dos relacionamentos intergeracionais e a saúde mental dos idosos. Pensando famílias, 18(1), 138-153.

Ramos, A., Stumpf Buaes, C. \& Doll, J. (2015). Apresentação. Educação e Envelhecimento. Educação e Realidade. Disponível em: http://www.redalyc.org. articulo.oa?id=317232811002

Reppold, C., Mayer, J., Almeida, L., \& Hutz, C. (2012). Avaliação da resiliência: controvérsia em torno do uso das escalas. Psicologia: Reflexão e Crítica, 25, 248-255.

Roksa, J., \& Potter, D. (2011). Parenting and academic achievement intergenerational transmission of educational advantage. Sociology of Education, 84 (4), 299-321. doi: $10.1177 / 0038040711417013$ 
Rutter, M. (2006). Implications of resilience concepts for scientific understanding. Annals of the New York Academy of Sciences, 1094, 1-12. doi: 10.1196/annals.1376.002

Ruwer, S., Rossi, A., \& Simon, L. (2005). Equilíbrio no idoso. Revista Brasileira de Otorrinolaringologia, 71, 298-303.

Santoro, M. (2014). Intergenerational solidarity between parents and adult children: a qualitative research among cohabiting and married children in Italy. International Review of Sociology: Revue Internationale de Sociologie, 24(3), 471-487. doi: 10.1080/03906701.2014.954334

Sarlet, I. (2001). Dignidade da Pessoa Humana e Direitos Fundamentais. Porto Alegre: Livraria do Advogado.

Silveira, M., Rocha, J., Vidmar, M., Wibelinger, L., \& Pasqualotti, A. (2010). Educação e inclusão digital para idosos. Revista Renole Novas Tecnologias na Educação, 8(2).

Tavares, W., Couto, G., \& Silva, R. (2012). Perfil das relações interpessoais e habilidades sociais de estudantes de psicologia. Estudos Interdisciplinares em Psicologia, 3(1), 75-92.

Tinajero, C., \& Páramo, M. (2012). The systems approach in developmental psychology: fundamental concepts and principles. Psicologia: Teoria e Pesquisa, 28(4), 457-465.

Uhlenberg, P. (2000). Integration of old and young. The Gerontologist, 40(3): 276-279. doi: 10.1093/geront/40.3.276

Yunes, M. (2003). Psicologia positiva e resiliência: o foco no indivíduo e na família. Psicologia em Estudo, 8 (num.esp.), 75-84.

Yunes, M. (2012). Psicologia Positiva e resiliência: o foco no indivíduo e na família, 8 (num. esp.) São Paulo: Casa do Psicólogo.

Windle, G., M Bennett, K. \& Noyes, J. (2011). A methodological review of resilience measurement scales. Health and Quality of Life Outcomes, 9(8), 1-18. doi: 10.1186/1477-7525-9-8

\section{Endereço para correspondência \\ Soniárlei Vieira Leite}

Universidade Salgado de Oliveira - UNIVERSO

Rua Marechal Deodoro 211, BI. B, 2o Andar, Centro, CEP 24030-060, Niterói - RJ, Brasil

Endereço eletrônico: soniarlei.vieira@gmail.com

\section{Lucia Helena de Freitas Pinho França}

Universidade Salgado de Oliveira - UNIVERSO

Rua Marechal Deodoro 211, BI. B, 2ㅇA마, Centro, CEP 24030-060, Niterói - RJ, Brasil

Endereço eletrônico: lucia.franca@gmail.com

Recebido em: 16/10/2015

Reformulado em: 08/11/2016

Aceito para publicação em: 18/11/2016 


\section{Notas}

* Docente de Graduação e Pós-Graduação da Universidade Veiga de Almeida, da Universidade Salgado de Oliveira e da Fundação Getúlio Vargas. Graduado em Direito pela Universidade do Estado do Rio de Janeiro (1990), especialista em Direito Tributário e em Direito do Estado pela Universidade Estácio de Sá (1992 e 1993), em Administração Financeira pela Fundação Getúlio Vargas (1995) e em Direito Educacional pela Pontifícia Universidade Católica (2010), mestre em Direito pela Universidade Estácio de Sá (2000) e doutor em Ciências Jurídicas e Sociais pela Universidad del Museo Social Argentino, AR (2013). Doutorando em Psicologia pela Universidade Salgado de Oliveira.

** Docente permanente do Programa de Pós-Graduação em Psicologia (Mestrado e Doutorado) da Universidade Salgado de Oliveira - Universo. Possui graduação em Psicologia pela Universidade Gama Filho (1978), especialização em Gerontologia Social pelo Instituto Sedes Sapientiae/SBGG-SP (1989), mestrado em Psicologia Social pela Universidade Federal do Rio de Janeiro (1989) e PhD em Psicologia pela University of Auckland, NZ (2004). Pesquisadora Bolsista Produtividade 2 CNPq 2014-2016. 